\title{
A NEW SURVEY ON BICLUSTERING OF MICROARRAY DATA
}

\author{
Haifa Ben Saber ${ }^{1,2}$ and Mourad Elloumi ${ }^{1,3}$ \\ ${ }^{1}$ Laboratory of Technologies of Information and Communication and Electrical \\ Engineering (LaTICE) at National Superior School of Engineers of Tunis \\ (ENSIT) - Tunis university, Tunis, Tunisia \\ ${ }^{2}$ Time université \\ ${ }^{3}$ University of Tunis-El Manar, Tunisia \\ ${ }^{2}$ bensaberhaifal@gmail.com, ${ }^{3}$ Mourad.Elloumi@gmail.com
}

\begin{abstract}
There are subsets of genes that have similar behavior under subsets of conditions, so we say that they coexpress, but behave independently under other subsets of conditions. Discovering such coexpressions can be helpful to uncover genomic knowledge such as gene networks or gene interactions. That is why, it is of utmost importance to make a simultaneous clustering of genes and conditions to identify clusters of genes that are coexpressed under clusters of conditions. This type of clustering is called biclustering.

Biclustering is an NP-hard problem. Consequently, heuristic algorithms are typically used to approximate this problem by finding suboptimal solutions. In this paper, we make a new survey on biclustering of gene expression data, also called microarray data.
\end{abstract}

\section{KEYWORDS}

Biclustering, heuristic algorithms, microarray data,genomic knowledge.

\section{INTRODUCTION}

A DNA Microarray is a glass slide covered with a chemical product and DNA samples containing thousands of genes. By placing this glass slide under a scanner, we obtain an image in which colored dots represent the expression level of genes under experimental conditions [1]. This process can be summerized by Figure 1. As shown in Figure 2, the obtained colored image can be coded by a matrix $M$, called gene expression data, or microarray data, where the $i^{\text {th }}$ row represents the $i^{\text {th }}$ gene, the $j^{\text {th }}$ column represents the $j^{\text {th }}$ condition and the cell $m_{i j}$ represents the expression level of the $i^{\text {th }}$ gene under the $j^{\text {th }}$ condition. Simultaneous clustering of rows (genes) and columns (conditions) of this matrix enables to identify subsets of genes that have similar behavior under subsets of conditions, so we say that they coexpress, but behave independently under other subsets of conditions. This type of clustering is called biclustering. Biclustering of microarray data can be helpful to discover coexpression of genes and, hence, uncover genomic knowledge such as gene networks or gene interactions. Biclustering is an NP-hard problem [3]. Consequently, heuristic algorithms are typically used to approximate this problem by finding suboptimal solutions. In this paper, we make a new survey on biclustering of microarray data.

Natarajan Meghanathan et al. (Eds) : NeTCoM, CSIT, GRAPH-HOC, SPTM - 2014

pp. 165-183, 2014. (C) CS \& IT-CSCP 2014

DOI : $10.5121 /$ csit.2014.41314 
In this paper, we make a survey on biclustering of gene expression data. The rest of the paper is organized as follows: First, we introduce some definitions related to biclustering of microarray data. Then, we present in section 3 some evaluation functions and biclustering algorithms. Next, we show how to validate biclusters via biclustering tools on microarrays datasets. Finally, we present our conclusion.
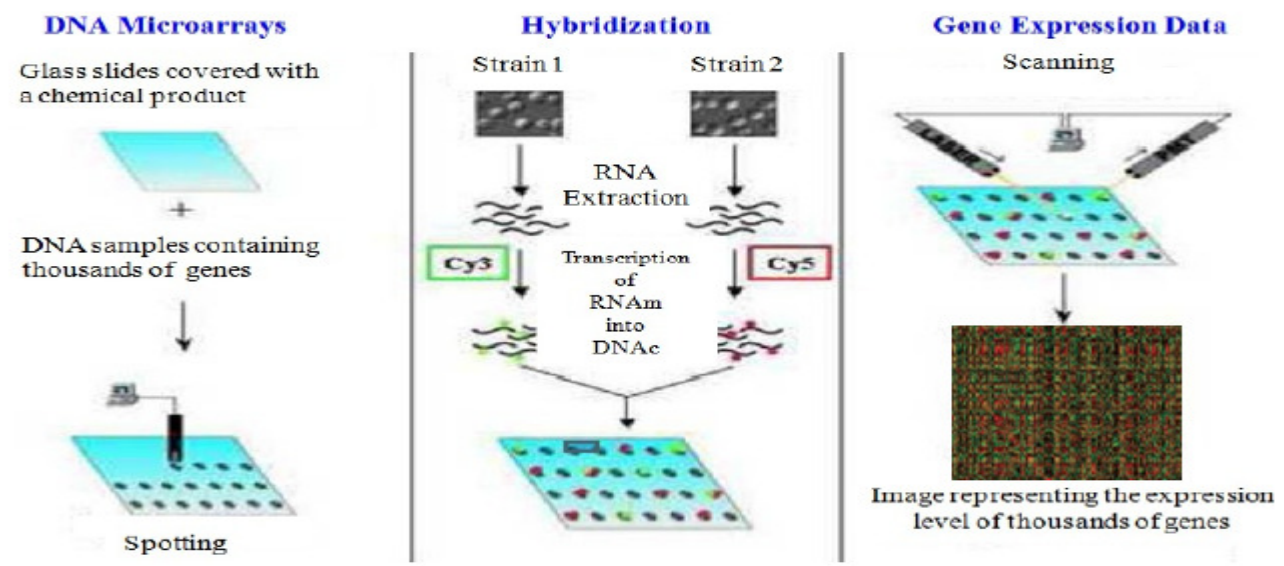

Figure 1. Generation from a DNA microarray of an image where colored dots represent the expression level of genes under experimental conditions [2]

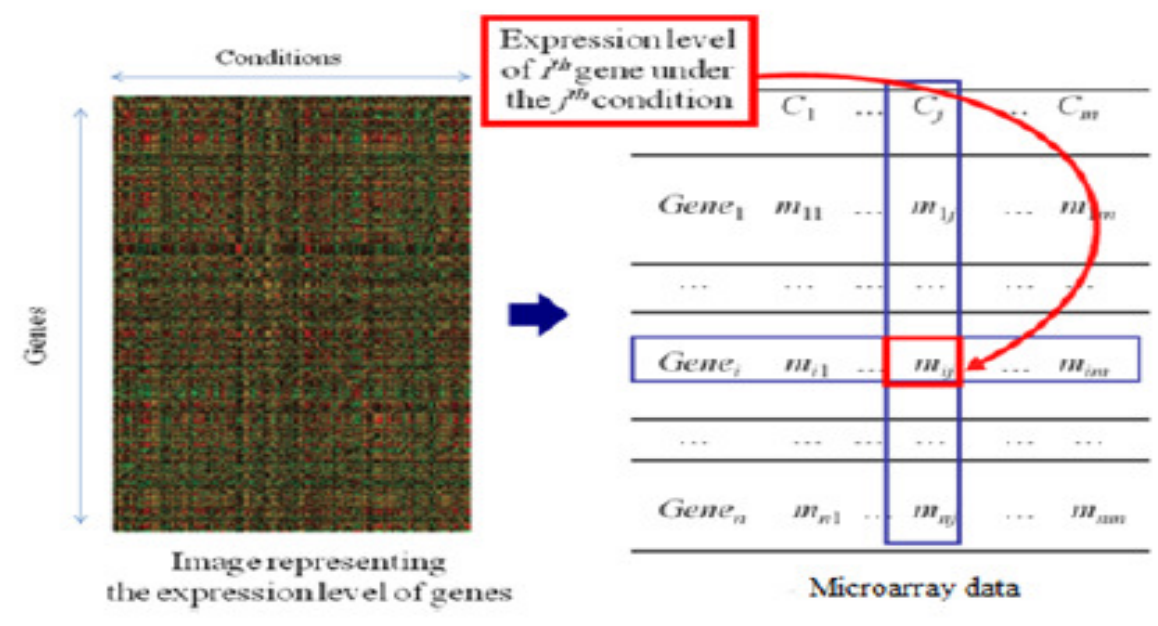

Figure 2. Coding of the generated colored image to a microarray data

\section{BICLUSTERING OF MICROARRAY DATA}

Let introduce some definitions related to a biclustering of microarray data [3].

Biclusters : Let $I=\{1,2, \ldots, n\}$ be a set of indices of $n$ genes, $I=\{1,2, \ldots, m\}$ be a set of indices of $m$ conditions and $M(I, J)$ be a data matrix associated with $I$ and $J$. A bicluster associated with the data matrix $M(I, J)$ is a couple $M\left(I^{\prime}, J^{\prime}\right)$ such that $I^{\prime} \subseteq I$ and $J^{\prime} \subseteq J$. 
Types of biclusters : A bicluster can be one of the following cases:

- Bicluster with constant values on rows:

$$
\begin{aligned}
& m_{i j}=c+a_{i} \\
& m_{i j}=c * a_{i}
\end{aligned}
$$

where $c$ is a constant and $a_{i}$ is the adjustment for the row $i$.

- Bicluster with constant values on columns:

$$
\begin{aligned}
& m_{i j}=c+b_{j} \\
& m_{i j}=c * b_{j}
\end{aligned}
$$

where $b_{j}$ is the adjustment for the column ${ }_{\mathrm{j}}$.

- Bicluster with coherent values: There are two types of biclusters with coherent values. Those with additive model and those with multiplicative model defined respectively by:

Those with additive model:

$$
m_{i j}=c+a_{i}+b_{j}
$$

And those with multiplicative model:

$$
m_{i j}=c * a_{i} * b_{j}
$$

- Bicluster with coherent evolution: It is a bicluster where all the rows (resp. columns) induce a linear order across a subset of columns (resp. rows).

Groups of biclusters : A group of biclusters can be one of the following types [4]:

1. Single bicluster (Figure 3. (a)),

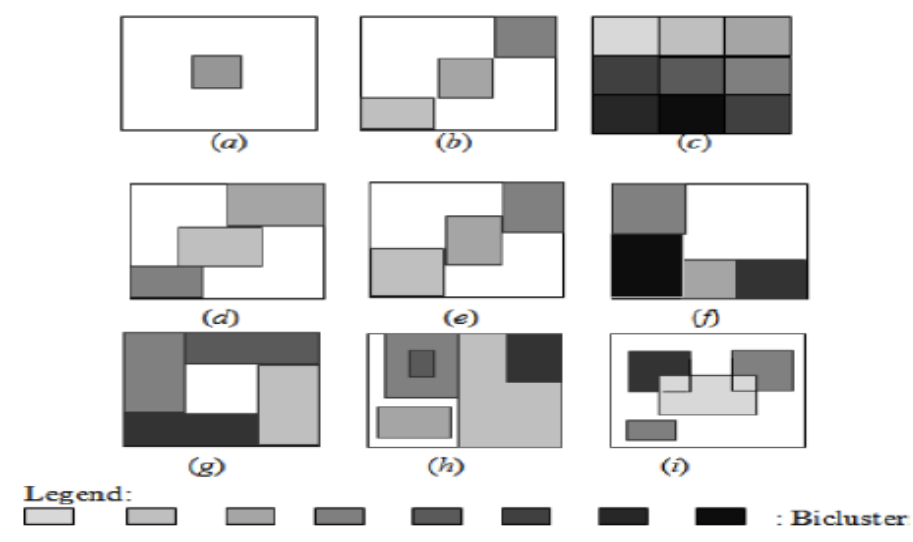

Figure 3.Types of groups of biclusters 
2. Exclusive rows and columns group of biclusters (Figure 3. (b)),

3. Non-overlapping group of biclusters with checkerboard structure (Figure 3. (c)),

4. Exclusive rows group of biclusters (Figure 3. (d)),

5. Exclusive columns group of biclusters (Figure 3. (e)),

6. Non-overlapping group of biclusters with tree structure (Figure 3. (f )),

7. Non-overlapping non-exclusive group of biclusters (Figure 3. (g)),

8. Overlapping group of biclusters with hierarchical structure (Figure 3. (h)),

9. Or, arbitrarily positioned overlapping group of biclusters (Figure 3. (i)).

We note also that a natural way to visualize a group of biclusters consists in assigning a different color to each bicluster and in reordering the rows and the columns of the data matrix so that we obtain a data matrix with colored blocks, where each block represents a bicluster.The biclustering problem can be formulated as follows: Given a data matrix $M$, construct a group of biclusters $B_{\text {opt }}$ associated with $M$ such that:

$$
f\left(B_{\text {opt }}\right)=\max _{B \in B C(M)} f(B)
$$

where $f$ is an objective function measuring the quality, i.e., degree of coherence, of a group of biclusters and $B C(M)$ is the set of all the possible groups of biclusters associated with $M$. This problem is NP-hard [4,5].

\section{EVALUATION FUNCTIONS}

An evaluation function is an indicator of the performance of a biclustering algorithm. There are two main classes of evaluation functions: Intra-biclusters evaluation functions and inter-biclusters evaluation functions.

\subsection{Intra-biclusters evaluation functions}

An intra-biclusters evaluation function is a function that measures the quality of a bicluster, i.e., it quantifies the coherence degree of a bicluster. There are several intra-biclusters evaluation functions.

- The $E_{A V S S}\left(I^{\prime}, J^{\prime}\right)$ is defined as follows[6]:

$$
E_{A V S S}\left(I^{\prime}, J^{\prime}\right)=\frac{\sum_{i \in I^{\prime}} \sum_{j \in J^{\prime}} s_{i j}}{\left|I^{\prime}\right|\left|J^{\prime}\right|}
$$

where $\left(I^{\prime}, J^{\prime}\right)$ is a bicluster, $s_{i j}$ is a similarity measure among elements of the row $i$ and the column $j$ with others elements belonging to $I^{\prime}$ and $J^{\prime}$. It follows that a number of these functions are particular cases of the AVerage Similarity Score (AVSS).

- The Average Row Variance (ARV) is defined as follows [7]:

$$
E_{A R V}\left(I^{\prime}, J^{\prime}\right)=\frac{\sum_{i \in I^{\prime}} \sum_{j \in J^{\prime}}\left(m_{i j}-m_{i J^{\prime}}\right)^{2}}{\left|I^{\prime}\right|\left|J^{\prime}\right|}
$$

where $m_{i J}$, is the average over the row $i$. It follows that the biclusters that contain rows with large changes in their values for different columns are characterized by a large row variance. The ARV guarantees that a bicluster captures rows exhibiting coherent trends under some subset columns. 
- The Mean Squared Residue (MSR) is defined as follows [8]:

$$
E_{M S R}\left(I^{\prime}, J^{\prime}\right)=\frac{\sum_{i \in I^{\prime}} \sum_{j \in J^{\prime}}\left(m_{i j}-m_{i J^{\prime}}-m_{I^{\prime} j}+m_{I^{\prime} J^{\prime}}\right)^{2}}{\left|I^{\prime}\right|\left|J^{\prime}\right|}
$$

where $m_{I^{\prime} J^{\prime}}$ is the average over the whole bicluster, $m_{I^{\prime} j}$ is the average over the column $j, m_{i J^{\prime}}$ is the average over the row $i$. The $E_{M S R}$ represents the variation associated with the interaction between the rows and the columns in the bicluster. It follows that a low (resp. high) $E_{M S R}$ value, i.e., close to 0 (resp. higher than a fixed threshold d), indicates that the bicluster is strongly (resp. weakly) coherent. The $E_{M S R}$ function is inadequate to assess certain types of biclusters. For example, the $E_{M S R}$ function is good for biclusters of coherent values with additive model but not for coherent values with multiplicative model.

- The Volume (V) is defined as follows [7]:

$$
E_{V}\left(I^{\prime}, J^{\prime}\right)=\left|I^{\prime}\right|\left|J^{\prime}\right|
$$

This function enables to have the maximum-sized bicluster that does not exceed a certain coherence value expressed as a MSR score. $E_{V}\left(I^{\prime}, J^{\prime}\right)$ finds the maximum-sized bicluster that does not exceed a certain coherence value [9] expressed as a MSR score. Hence, discovered biclusters have a high $E_{V}\left(I^{\prime}, J^{\prime}\right)$ maximized and lower $E_{M S R}$ than a given threshold $\delta \geq 0$.

- The Mean Square Error (MSE) is defined as follows [10]:

$$
E_{M S E}(I, J)=\frac{\sum_{i \in I} \sum_{j \in J}\left(m_{i j}-m_{i J^{\prime}}-m_{I j}+m_{I J}\right)^{2}}{|I||J|}
$$

where $m_{I J}$ is the average over the whole matrix, $m_{I j}$ is the average over the column $j$ of the whole matrix and $m_{i J}$ is the average over the row $i$. This function identifies constant biclusters.

- The Average Correlation Value (ACV) is defined as follows [5, 11]:

$$
E_{A C V}\left(I^{\prime}, J^{\prime}\right)=\max \left\{\begin{array}{ll}
\sum_{i \in I^{\prime}} \sum_{j \in I^{\prime}}\left|r_{i j}\right|-\left|I^{\prime}\right| & \sum_{k \in J^{\prime}} \sum_{l \in J^{\prime}}\left|r_{k l}\right|-\left|J^{\prime}\right| \\
\left|I^{\prime}\right|\left(\left|I^{\prime}\right|-1\right) & \}\left(J^{\prime} \mid-1\right)
\end{array}\right\}
$$

where $r_{i j}(i \neq j)$ (resp. $r_{k l}(k \neq l)$ ) is the Pearson's correlation coefficient associated with the row indices $i$ and $j$ (resp. $k$ and $l$ ) in the bicluster $\left(J^{\prime}, J^{\prime}\right)$ [8]. The values of $E_{A C V}$ belong to [0;1], hence, a high (resp. low) $E_{A C V}$ value, i.e., close to 1 (resp. close to 0), indicates that the bicluster is strongly (resp. weakly) coherent. However, the performance of the $E_{A C V}$ function decreases when noise exists in the data matrix $[5,11]$.

- The Average Spearman's Rho (ASR) is defined as follows [2]:

$$
E_{A S R}\left(I^{\prime}, J^{\prime}\right)=2 \max \left\{\frac{\sum_{i \in I^{\prime}} \sum_{j \in I^{\prime}, j \geq i+1} \rho_{i j}}{\left|I^{\prime}\right|\left(\left|I^{\prime}\right|-1\right)}, \frac{\sum_{k \in J^{\prime}} \sum_{l \in J^{\prime}, I \geq k+1} \rho_{k l}}{\left|J^{\prime}\right|\left(\left|J^{\prime}\right|-1\right)}\right\}
$$

where $\rho_{i j}(i \neq j)$ (resp. $\rho_{K L}(k \neq l)$ ) is the Spearman's rank correlation associated with the row indices $i$ and $j$ in the bicluster $\left(I^{\prime}, J^{\prime}\right)[12]$, The values of the $E_{A S R}$ function belong also to [-1,1], 
hence, a high (resp. low) $E_{A S R}$ value, i.e., close to 1 (resp. close to -1), indicates that the bicluster is strongly (resp. weakly) coherent. On the other hand, like Spearman's rank correlation, the $E_{A S R}$ is less sensitive to the presence of noise in data [2]. There are other intra-biclusters evaluation function like the Average Correspondance Similarity Index (ACSI) [2].

\subsection{Inter-biclusters evaluation functions}

An inter-biclusters evaluation function is a function that measures the quality of a group of biclusters, i.e., it assesses the accuracy of an algorithm to recover true implanted biclusters in a data matrix. There are several inter-biclusters evaluation functions. In what follows, we present some of them:

Let $M_{1}$ and $M_{2}$ be two groups of biclusters defined as follows:

$M_{1}=\left\{B_{1}^{(1)}, B_{2}^{(1)}, \ldots, B_{K_{1}}^{(1)}\right\}$, where $B_{l}^{(1)}=\left(G_{l}^{(1)}, C_{l}^{(1)}\right), G_{l}$ and $C_{l}$ are respectively the $l^{\text {th }}$ gene and condition, $1 \leq l \leq K_{1}$ : Set of true implanted biclusters in a data matrix $M$.

$M_{2}=\left\{B_{1}^{(2)}, B_{2}^{(2)}, \ldots, B_{K_{2}}^{(2)}\right\}$, where $B_{m}^{(j)}=\left(G_{m}^{(2)}, C_{m}^{(2)}\right), G_{m}$ and $C_{m}$ are respectively the $m^{\text {th }}$ gene and condition, $1 \leq m \leq K_{2}$ : Set of the biclusters extracted by a biclustering algorithm.

- The Prelic index is defined as follows:

$$
I_{\text {relic }}\left(M_{1}, M_{2}\right)=\frac{1}{K_{1}} \sum_{i=1}^{n_{1}} \max _{j} S_{P_{\text {relic }}}\left(B_{i}^{(1)}, B_{j}^{(2)}\right)
$$

where $S_{\text {Prelic }}$ is based on the Jaccard index for two sets and defined as follows:

$$
S_{P_{\text {relic }}}\left(B_{i}, B_{j}\right)=\frac{\left|G_{i} \cap G_{j}\right|}{\left|G_{i} \cup G_{j}\right|}
$$

This index compares two solutions based on categorization of genes. However, it compares only genes sets.

- The Liu and Wang index is defined as follows:

$$
I_{\text {Liu\&Wang }}\left(M_{1}, M_{2}\right)=\frac{1}{K_{1}} \sum_{i=1}^{K_{1}} \max _{j} S_{\text {Liu\&Wang }}\left(B_{i}^{(1)}, B_{j}^{(2)}\right)
$$

where

$$
S_{\text {Liu\&Wang }}\left(B_{i}, B_{j}\right)=\frac{\left|G_{i} \cap G_{j}\right|+\left|C_{i} \cap C_{j}\right|}{\left|G_{i} \cup G_{j}\right|+\left|C_{i} \cup C_{j}\right|}
$$

It compares two solutions by considering both genes and conditions.

- The wtjaccard index is defined as follows:

$$
=\frac{\sum_{i=1}^{K_{1}}\left|B_{i}^{(1)}\right| * \max _{j} S_{\text {Jaccard }}\left(B_{i}^{(1)}, B_{j}^{(2)}\right)}{\sum_{i}^{K_{1}}\left|B_{i}^{(1)}\right|}
$$


Where

$$
S_{\text {Jaccard }}\left(B_{i}, B_{j}\right)=\frac{\left|C_{i} \cap B_{j}\right|+\left|G_{i} \cap G_{j}\right|}{\left|C_{i}\right|+\left|B_{j}\right|-\left|C_{i} \cap C_{j}\right|}
$$

- The Dice index is defined as follows:

$$
I_{\text {Dice }}\left(M_{1}, M_{2}\right)=\frac{1}{K_{1}} \sum_{i=1}^{K_{1}} \max _{j} S_{\text {Dice }}\left(B_{i}^{(1)}, B_{j}^{(2)}\right)
$$

where:

$$
S_{\text {Dice }}\left(B_{i}, B_{j}\right)=\frac{2 *\left|C_{i} \cap C_{j}\right|}{\left|C_{i}\right|+\left|C_{j}\right|}
$$

which is proposed in [13] and called F-measure in biclustering cases to computes the overall relevance of two bicluster solutions.

- The Santamaría index is defined as follows:

$$
I_{\text {wtDice }}\left(M_{1}, M_{2}\right)=\frac{\sum_{i=1}^{K_{1}}\left|B_{i}^{(1)}\right| * \max _{j} S_{\text {Dice }}\left(B_{i}^{(1)}, B_{j}^{(2)}\right)}{\sum_{i=1}^{K_{1}}\left|B_{i}^{(1)}\right|}
$$

The Santamaría index is the most conservative index among above others indices and used for biclustering case $[14,13]$. In fact, while the Prelic index compares only object sets and the LW index compares object sets and feature sets independently, the Santamaría index compares two solutions using pairs of genes and conditions.

For gene expression case, the Gene Match Score (GMS) function doesn't take into account column match. It is given by:

$$
E_{G M S}\left(B_{1}, B_{2}\right)=\frac{1}{\left|B_{1}\right|} \sum_{\left(I_{1}, J_{1}\right) \in B_{1}} \max _{\left(I_{2}, J_{2}\right) \in B_{2}} \frac{\left|I_{1} \cap I_{2}\right|}{\left|I_{1} \cup I_{2}\right|},
$$

where $B_{1}$ and $B_{2}$ are two groups of biclusters and the pair $(I, J)$ represents the submatrix whose rows and columns are given by the set $I$ and $J$, respectively.

The Row and Column Match Scores (RCMS) assess the method's accuracy to recover known biclusters and reveal true ones. Thereafter, more similar measures of match scores have been introduced [5, 15, 6]. For instance, the evaluation functions, herein called Row and Column Match Scores, $E_{R C M S 1}$ and $E_{R C M S 2}$, are proposed in [6] and [15], respectively and given by:

$$
\begin{aligned}
& E_{R C M S_{1}}\left(B_{1}, B_{2}\right)=\frac{1}{\left|B_{1}\right|} \sum_{\left(I_{1}, J_{1}\right) \in B_{1}} \max _{\left(I_{2}, J_{2}\right) \in B_{2}} \frac{\left|I_{1} \cap I_{2}\right|+\left|J_{1} \cap J_{2}\right|}{\left|I_{1} \cup I_{2}\right|+\left|J_{1} \cup J_{2}\right|}, \\
& E_{R C M S_{2}}\left(B_{1}, B_{2}\right)=\frac{1}{\left|B_{1}\right|} \sum_{\left(I_{1}, J_{1}\right) \in B_{1}} \max _{\left.I_{2}, J_{2}\right) \in B_{2}} \frac{\left|I_{1} \cap I_{2}\right|+\left|J_{1} \cap J_{2}\right|}{\left|I_{1}\right|+\left|J_{1}\right|}
\end{aligned}
$$


All these measures of match score are used to assess the accuracy of an algorithm to recover known biclusters and reveal true ones. Both $E_{R C M S 1}$ and $E_{R C M S 2}$ have the advantage of reflecting, simultaneously, the match of the row and column dimensions between biclusters as opposed to $E_{G M S}$ that doesn't take into account column match. They vary between 0 and 1 (the higher the better the accuracy). Let $B_{o p t}$ denote the set of true implanted biclusters in the data matrix $M$ and $B$ the set of the output biclusters of a biclustering algorithm. Thus, $E_{G M S}\left(B_{o p t}, B\right)$ and $E_{R C M S I}\left(B_{o p t}, B\right)$ express how well each of the true biclusters are detected by the algorithm under consideration. $E_{R C M S 2}\left(B_{X}, B_{Y}\right)$, where $B_{X}$ (resp. $\left.B_{Y}\right)$ denotes the set of biclusters detected by the algorithm $X$ (resp. Algorithm $Y$ ), has the particularity to allow the quantification of how well each bicluster identified by the algorithm $X$ is contained into some bicluster detected by the algorithm $Y$.

\section{BICLUSTERING ALGORITHMS}

As we mentioned earlier, the biclustering problem is NP-hard [3, 10]. Consequently, heuristic algorithms are typically used to approximate the problem by finding suboptimal solutions. We distinguish different approaches adopted by biclustering approaches[3].

\subsection{Iterative Row and Column Clustering Combination Approach}

By adopting the Iterative Row and Column Clustering Combination Approach (IRCCC) approach, we apply clustering algorithms on both rows and columns separately and then combine the results to obtain biclusters [56]. Table 5 is a synoptic table of biclustering algorithms adopting IRCCC approach. The conceptually simpler way to perform biclustering using existing algorithms without searching novels algorithms. But, this approach consider approximatively same advantages and drawbacks that clustering algorithms used. Among the algorithms adopting this approach we mention Croki2 [58], Crobin [58], DCC [59], ITWC [61], CTWC [54] and BiSOM [60].

Table 1. Biclustering algorithms adopting IRCCC approach.

\begin{tabular}{|l|l|l|l|c|}
\hline $\begin{array}{l}\text { Algorithms } \\
\text { Bicluster } \\
\text { discovery }\end{array}$ & $\begin{array}{l}\text { Types of } \\
\text { biclusters }\end{array}$ & $\begin{array}{l}\text { Types of groups } \\
\text { of biclusters }\end{array}$ & Data type & Time complexity \\
\hline Croeuc [57] & Coherent values & - & $\begin{array}{l}\text { One at time } \\
\text { Continuous }\end{array}$ & - \\
\hline Croki2 [58] & Coherent values & - & $\begin{array}{l}\text { One at time } \\
\text { Continuous }\end{array}$ & - \\
\hline CroBin[57] & Coherent values & - & $\begin{array}{l}\text { One at time } \\
\text { Continuous }\end{array}$ & - \\
\hline CemCroki [57] & Coherent values & - & $\begin{array}{l}\text { One at time } \\
\text { Continuous }\end{array}$ & - \\
\hline DCC [59] & Coherent values & $\begin{array}{l}\text { Exclusive } \\
\text { dimension }\end{array}$ & $\begin{array}{l}\text { One at time } \\
\text { Continuous }\end{array}$ & - \\
\hline Bi-SOM [60] & Coherent values & - & $\begin{array}{l}- \\
\text { One at time } \\
\text { Continuous }\end{array}$ & - \\
\hline ITWC [61] & Coherent values & \multicolumn{1}{|c|}{$\begin{array}{l}\text { One at time } \\
\text { Continuous }\end{array}$} & - \\
\hline CTWC[54] & Constant columns & $\begin{array}{l}\text { Arbitrarily } \\
\text { positioned } \\
\text { overlapping }\end{array}$ & \multicolumn{2}{|}{} \\
\hline
\end{tabular}




\subsection{Greedy Iterative Search Approach}

By adopting the Greedy Iterative Search (GIS), first, we construct submatrices of the data matrix by adding/removing a row/column to/from the current submatrix that optimizes a certain function. Then, we reiterate this process until no other row/column can be added / removed to/from any submatrix. This approach presents the same advantage and drawback as DC. They may make wrong decisions and loose good biclusters, but they have the potential to be very fast. Among the algorithms adopting this approach we mention Spectral [16], Quest [17], Random Walk Biclustering [18], BicFinder [19], MSB [6], ISA [17, 20], OPSM [21] and SAMBA [17, 22]. Table 1 is a synoptic table of biclustering algorithms adopting GIS approach.

Table 2. Biclustering algorithms adopting GIS approach.

\begin{tabular}{|c|c|c|c|c|c|}
\hline Algorithms & $\begin{array}{l}\text { Types of } \\
\text { biclusters }\end{array}$ & $\begin{array}{l}\text { Types of groups } \\
\text { of biclusters }\end{array}$ & $\begin{array}{l}\text { Bicluster } \\
\text { discover } \\
\text { y } \\
\text { strategy }\end{array}$ & Data type & Time complexity \\
\hline d-biclusters[10] & $\begin{array}{l}\text { Coherent } \\
\text { values }\end{array}$ & $\begin{array}{l}\text { Arbitrarily } \\
\text { positioned } \\
\text { overlapping }\end{array}$ & $\begin{array}{l}\text { One at a } \\
\text { time }\end{array}$ & Continuous & $O(n m)$ \\
\hline FLOC [23] & $\begin{array}{l}\text { Coherent } \\
\text { values }\end{array}$ & $\begin{array}{l}\text { Arbitrarily } \\
\text { positioned } \\
\text { overlapping }\end{array}$ & $\begin{array}{l}\text { All at } \\
\text { time }\end{array}$ & Continuous & $O\left((n+m)^{2} k p\right.$ \\
\hline xMotif [17] & $\begin{array}{l}\text { Coherent } \\
\text { evolution }\end{array}$ & $\begin{array}{l}\text { Single bicluster } \\
\text { arbitrarily } \\
\text { positioned } \\
\text { overlapping }\end{array}$ & $\begin{array}{l}\text { All at } \\
\text { time }\end{array}$ & Discret & - \\
\hline RMSBE [8] & $\begin{array}{l}\text { Constant } \\
\text { values }\end{array}$ & - & $\begin{array}{l}\text { All at } \\
\text { time }\end{array}$ & Binary & $O\left(k C_{u}\left(1-p_{r}\right)\left((n+m)+p_{r}\right)\right)$ \\
\hline MSB[6] & $\begin{array}{l}\text { Constant } \\
\text { values }\end{array}$ & - & $\begin{array}{l}\text { All at } \\
\text { time }\end{array}$ & Binary & $O\left((n+m)^{2}\right) O\left(k\left(n^{2}+m^{2}\right)\right)$ \\
\hline OPSMs [24] & $\begin{array}{l}\text { Coherent } \\
\text { evolution }\end{array}$ & $\begin{array}{l}\text { Single bicluster } \\
\text { arbitrarily } \\
\text { positioned } \\
\text { overlapping }\end{array}$ & $\begin{array}{l}\text { One at a } \\
\text { time }\end{array}$ & Continuous & $O\left(n m^{3} I\right)$ \\
\hline Spectral [16] & $\begin{array}{l}\text { Coherent } \\
\text { values }\end{array}$ & $\begin{array}{l}\text { Checkerboard } \\
\text { structure }\end{array}$ & $\begin{array}{l}\text { All at } \\
\text { time }\end{array}$ & Continuous & - \\
\hline $\begin{array}{l}\text { d-Pattern[17, } \\
10]\end{array}$ & $\begin{array}{l}\text { Constant } \\
\text { rows } \\
\text { values }\end{array}$ & $\begin{array}{l}\text { Arbitrarily } \\
\text { positioned } \\
\text { overlapping }\end{array}$ & $\begin{array}{l}\text { All at } \\
\text { time }\end{array}$ & Continuous & $O(n m(n+m) k)$ \\
\hline BISOFT[25] & $\begin{array}{l}\text { Coherent } \\
\text { values }\end{array}$ & & $\begin{array}{l}\text { One at a } \\
\text { time }\end{array}$ & Categorical & - \\
\hline sv4d [26] & $\begin{array}{l}\text { Constant } \\
\text { values }\end{array}$ & $\begin{array}{l}\text { A checkerboard } \\
\text { structure }\end{array}$ & $\begin{array}{l}\text { All at } \\
\text { time }\end{array}$ & Binary & - \\
\hline ISA[17] & $\begin{array}{l}\text { Coherent } \\
\text { values }\end{array}$ & Overlapping & $\begin{array}{l}\text { One at } \\
\text { time }\end{array}$ & Continuous & - \\
\hline BicBin [27] & $\begin{array}{l}\text { Constant } \\
\text { values }\end{array}$ & Overlapping & $\begin{array}{l}\text { A set of } \\
\text { biclusters }\end{array}$ & Binary & - \\
\hline
\end{tabular}

where :

$n$ and $m$ are respectively the numbers of genes and conditions in the data matrix, $l$ is the number of the best partial models of order, 
$K$ is the maximum number of iterations,

$C_{u}$ isthe cost of computing the new residue and the new row variance of the bicluster after performing a move,

$p_{r}$ is a user-provided probability that the algorithm is allowed to execute a random move.

\subsection{Exhaustive Bicluster Enumeration Approach}

By adopting the Exhaustive Bicluster Enumeration (EBE), We identify all the possible groups of biclusters in order to keep the best one, i.e., the one that optimizes a certain evaluation function. The advantage of this approach is that it is able to obtain the best solutions. Its drawback is that it is costly in computing time and memory space Among the algorithms adopting this approach we mention BSGP[28, 29], OPC [30, 6], CPB [30], IT[31], e-Bmotif [29], BIMODULE [32], RAP [26], BBK [33] and MSB [6]. Table 2 is a synoptic table of biclustering algorithms adopting EBE approach.

Table 3. Biclustering algorithms adopting EBE approach.

\begin{tabular}{|c|c|c|c|c|c|}
\hline $\begin{array}{l}\text { Algorith } \\
\text { ms }\end{array}$ & $\begin{array}{l}\text { Types of } \\
\text { biclusters }\end{array}$ & $\begin{array}{l}\text { Types of } \\
\text { groups of } \\
\text { biclusters }\end{array}$ & $\begin{array}{l}\text { Bicluster } \\
\text { discovery } \\
\text { strategy }\end{array}$ & Data type & Time complexity \\
\hline $\begin{array}{l}\text { e-BiMotif } \\
{[34][29]}\end{array}$ & Coherent values & - & All at time & Contingence & $O\left(2^{n} m \log (m)\right)$ \\
\hline CPB [30] & Coherent values & - & All at time & $\begin{array}{l}\text { Contingence } \\
\text { Categorical }\end{array}$ & - \\
\hline OPC [30] & $\begin{array}{l}\text { Coherent } \\
\text { evolution }\end{array}$ & $\begin{array}{l}\text { Arbitrarily } \\
\text { positioned } \\
\text { overlapping }\end{array}$ & All at time & & - \\
\hline $\begin{array}{l}\text { PClusters } \\
{[10]}\end{array}$ & Coherent values & $\begin{array}{l}\text { Non- } \\
\text { overlapping } \\
\text { non-exclusive }\end{array}$ & All at time & Binary & $O\left(n^{2} m^{4}(n \log (n)+m \log (m))\right)$ \\
\hline $\begin{array}{l}\text { BSGP } \\
{[28,29]}\end{array}$ & Coherent values & - & All at time & $\begin{array}{l}\text { Contingence } \\
\text { Categorical }\end{array}$ & - \\
\hline $\begin{array}{l}\text { Expander } \\
\text { [35] }\end{array}$ & $\begin{array}{l}\text { Coherent } \\
\text { evolution }\end{array}$ & - & One a time & Categorical & - \\
\hline IT [31] & Coherent values & - & All at time & Contingence & - \\
\hline $\begin{array}{l}\text { BIMODU } \\
\text { LE [32] }\end{array}$ & Coherent values & - & One a time & $\begin{array}{l}\text { Contingence } \\
\text { Categorical }\end{array}$ & - \\
\hline RAP [26] & $\begin{array}{l}\text { Constant row } \\
\text { values coherent } \\
\text { values }\end{array}$ & Overlapping & One a time & Continuous & - \\
\hline $\begin{array}{l}\text { SAMBA } \\
{[17,22]}\end{array}$ & $\begin{array}{l}\text { Coherent } \\
\text { evolution }\end{array}$ & $\begin{array}{l}\text { Arbitrarily } \\
\text { positioned } \\
\text { Overlapping }\end{array}$ & All at time & Continuous & $O\left(\left(n 2^{d+1}\right)^{\log (r+1) / r(r d)}\right)$ \\
\hline $\operatorname{MDS}[36]$ & & - & & & $O\left(2^{m}+m^{2} n \log (n)+n^{2} m \log (m)\right)$ \\
\hline $\begin{array}{l}\text { cHawk } \\
{[37]}\end{array}$ & $\begin{array}{l}\text { Constant } \\
\text { values//coherent } \\
\text { Evolution }\end{array}$ & Overlapping & All at time & Categorial & - \\
\hline BBK[33] & Constant values & - & One at time & Binary & - \\
\hline
\end{tabular}

Where

$d$ is the bounded degree of gene vertices in a bipartite graph $G$ whose two sides correspond to he set of genes and the set of conditions.

$r$ is the maximum weight edge in the bipartite graph $G$. 


\subsection{Distribution Parameter Identification Approach}

By adopting the Distribution Parameter Identification (DPI) approach use a statistical model to identify the distribution parameters and generate the data by minimizing a certain criterion iteratively. These algorithms certainly find the best biclusters, if they exist, but have a very serious drawback. Due to their high complexity, they can only be executed by assuming restrictions on the size of the biclusters. Among the algorithms adopting this approach we mention QUBIC [38], PRMs [39], FABIA [40], BEM [41] and BCEM [42]. Table 3 is a synoptic table of biclustering algorithms adopting DPI approach.

Table 4. Biclustering algorithms adopting DPI approach.

\begin{tabular}{|c|c|c|c|c|c|}
\hline $\begin{array}{l}\text { Algorithms } \\
\text { Bicluster } \\
\text { discovery }\end{array}$ & $\begin{array}{l}\text { Types of } \\
\text { biclusters }\end{array}$ & $\begin{array}{l}\text { Types of } \\
\text { groups of } \\
\text { biclusters }\end{array}$ & $\begin{array}{l}\text { Bicluster } \\
\text { discovery } \\
\text { strategy }\end{array}$ & Data type & $\begin{array}{l}\text { Time } \\
\text { complexity }\end{array}$ \\
\hline PRMs [43] & $\begin{array}{l}\text { Coherent } \\
\text { constant } \\
\text { values on } \\
\text { Columns }\end{array}$ & $\begin{array}{l}\text { Arbitrarily } \\
\text { positioned } \\
\text { overlapping }\end{array}$ & All at time & Binary & - \\
\hline iBBiG[44] & $\begin{array}{c}\text { Coherent } \\
\text { values }\end{array}$ & Overlapping & $\begin{array}{l}\text { One set at } \\
\text { time Binary }\end{array}$ & Binary & - \\
\hline Plaid $[45,46]$ & $\begin{array}{l}\text { Coherent } \\
\text { values }\end{array}$ & $\begin{array}{c}\text { Arbitrarily } \\
\text { positioned } \\
\text { overlapping }\end{array}$ & One at time & Continuous & $O\left(n^{2}\right)$ \\
\hline QUBIC[38] & $\begin{array}{l}\text { Constant } \\
\text { columns or } \\
\text { rows }\end{array}$ & $\begin{array}{l}\text { Exclusive } \\
\text { dimension }\end{array}$ & One at time & Discrete & - \\
\hline FABIA[40] & $\begin{array}{c}\text { Constant } \\
\text { values }\end{array}$ & Overlapping & All at time & $\begin{array}{c}\text { Catgeorial } \\
\text { binary }\end{array}$ & - \\
\hline BEM [41] & $\begin{array}{l}\text { Coherent } \\
\text { values }\end{array}$ & - & All at time & $\begin{array}{c}\text { Continuous } \\
\text { binary }\end{array}$ & $O(\mathrm{~nm})$ \\
\hline BCEM[42] & $\begin{array}{c}\text { Coherent } \\
\text { values }\end{array}$ & - & All at time & $\begin{array}{c}\text { Continuous } \\
\text { binary }\end{array}$ & - \\
\hline ISA [20] & $\begin{array}{c}\text { Coherent or } \\
\text { constant } \\
\text { values } \\
\end{array}$ & - & $\begin{array}{l}\text { One at a } \\
\text { time }\end{array}$ & Continuous & - \\
\hline Gibbs[47] & $\begin{array}{l}\text { Constant } \\
\text { columns or } \\
\text { rows }\end{array}$ & $\begin{array}{l}\text { Exclusive } \\
\text { dimension }\end{array}$ & $\begin{array}{l}\text { One at a } \\
\text { time }\end{array}$ & $\begin{array}{c}\text { Catgeorial } \\
\text { binary }\end{array}$ & - \\
\hline
\end{tabular}

\subsection{Divide and Conquer Approach}

By adopting the Divide-and-Conquer (DC) approach, first, we start by a bicluster representing the whole data matrix then we partition this matrix in two submatrices to obtain two biclusters. Next, we reiterate recursively this process until we obtain a certain number of biclusters verifying a specific set of properties. The advantage of DC is that it is fast, its drawback is that it may ignore good biclusters by partitioning them before identifying them. DC algorithms have the significant advantage of being potentially very fast. However, they have the very significant drawback of being likely to miss good biclusters that may be split before they can be identified. Among the algorithms adopting this approach we mention OWS [48], TWS [49], BiBit [28] and BARTMAP [50] and GS [51]. 
Table 5. Biclustering algorithms adopting DC approach.

\begin{tabular}{|c|c|c|c|c|}
\hline $\begin{array}{l}\text { Algorithms } \\
\text { Bicluster } \\
\text { discovery }\end{array}$ & $\begin{array}{l}\text { Types of } \\
\text { biclusters }\end{array}$ & $\begin{array}{l}\text { Types of groups } \\
\text { of biclusters }\end{array}$ & Data type & Time complexity \\
\hline $\begin{array}{c}\text { Block Clustering } \\
\text { [52] }\end{array}$ & $\begin{array}{l}\text { Constant } \\
\text { values }\end{array}$ & $\begin{array}{l}\text { Non-overlapping } \\
\text { tree structure }\end{array}$ & $\begin{array}{l}\text { Binary } \\
\text { categorial }\end{array}$ & - \\
\hline OWS[53] & $\begin{array}{l}\text { Constant } \\
\text { values }\end{array}$ & All at time & Continuous & $O(n)$ \\
\hline TWS [54] & $\begin{array}{l}\text { Constant } \\
\text { values }\end{array}$ & All at time & Continuous & - \\
\hline BiBit [28] & $\begin{array}{l}\text { Constant } \\
\text { values }\end{array}$ & All at time & Binary & $O(n m \beta \min \{n, m\})$ \\
\hline BiBit [28] & $\begin{array}{l}\text { Constant } \\
\text { values }\end{array}$ & All at time & Binary & - \\
\hline Cmnk [44] & $\begin{array}{l}\text { Constant } \\
\text { values }\end{array}$ & One at time & Binary & - \\
\hline GS [51] & $\begin{array}{l}\text { Constant } \\
\text { values }\end{array}$ & One at time & Binary & - \\
\hline
\end{tabular}

where $\beta$ is the number of biclusters that are not entirely contained in any other bicluster.

\section{BICLUSTERING VALIDATION}

There are two types of biclusters validation;

(i) Statistical validation: It is used to validate synthetical data

(ii) Biological validation: It is used to validate biological data

\subsection{Statistical validation}

Statistical validation can be made by adopting one or many of the following indices:

Separation: It reflects how well the biclusters are separated from each other. Separation between two biclusters

$\mathrm{A}$ and $\mathrm{B}$ is defined as follows [62]:

$$
\operatorname{Sep}(A, B)=1-\frac{A \cap B}{A \cup B}
$$

Coverage: We distinguish three types of coverage, matrix coverage, genes coverage and conditions coverage:

$$
\begin{gathered}
\text { Matrix coverage }=\frac{\text { Number of the cells covered by the extracted biclusters }}{\text { Total number of cells in the mat rix }} \\
\text { Genes coverage }=\frac{\text { Number of the genes covered by the extracted biclusters }}{\text { Total number of genes in the matrix }} \\
\text { Conditions coverage }=\frac{\text { Number of the conditions covered by the extracted biclusters }}{\text { Total number of conditions in the mat rix }}
\end{gathered}
$$


Compactness: It assesses cluster homogeneity, with intra-cluster variance[63].

Connectedness: It assesses how well a given partitioning groups data items together with their nearest neighbours in the data space [63].

Coherence: It expresses how well a bicluster is fitted to a specified model. The coherence is computed thanks to compactness and connectedness.

Significance: It is computed thanks to $p$-value ${ }_{B}$. Let $\mathrm{B}$ be a bicluster, $\mathrm{p} \square$ value is defined as follows [15]:

$$
p \text {-value }{ }_{B}=1-\phi\left(\frac{\left|1_{B}\right| /|B|-p}{\sqrt{\frac{p(1-p)}{|B|}}}\right)
$$

where $\mathrm{f}$ is the standard normal distribution function, $\left|1_{\mathrm{B}}\right|$ is the number of 1 's in the bicluster $B$ and $p=k /\left(|I|^{*}|J|\right)$ of 1's in $M(I, J), k$ is the number of 1 's in the binary matrix $M_{b}$. A bicluster $B$ is considered as potentially significant at a level of significance $\alpha$ if $p$-value ${ }_{B}<\alpha$.

\subsection{Biological validation}

Biological validation can qualitatively evaluate the capacity of an algorithm to extract meaningful biclusters from a biological point of view. To assess biologically biclusters, we can use Gene Ontology (GO) annotation [64]. In GO, genes are assigned to three structured, controlled vocabularies, called ontologies: biological process, cellular components and molecular functions. The GO Consortium (GOC)[64] [65] is involved in the development and application of the GO. In what follows, we briefly report some R tools relared to GOC [66, 67]:

- AnnotationDbi: It provides user interface and database connection code for annotation data packages using SQLite data storage.

- FunCluster: It is a functional profiling and analysis of microarray expression data based on GO \& KEGG.

- GExMap: It is an intuitive visual tool to perform a GO and to test to unveil genomic clusters, graphical interpretations and statistical results in pdf files.

- GO.db annotation: It provides detailed information about the latest version of the GOs and it is updated biannually.

- GOsummaries: It shows GO enrichment results in the context of experimental data.

- GOstats: It determines which GOs found in gene lists are statistically over/underrepresented.

- goTools: It compares the GOs represented by the genes in the three gene lists (biological process, molecular function and cellular component).

- topGO: It provides tools for testing GO terms while accounting for the topology of the GO graph. Different test statistics and different methods for eliminating local similarities and dependencies between GO terms can be implemented and applied.

\section{TOOLS}

There are also many $\mathrm{R}$ microarray biclustering tools. Table 6 . presents a few examples on tools and here are some examples [68]: 
- arules: It is a mining association rules and frequent itemsets. It provides the infrastructure for representing, manipulating and analyzing transaction data and patterns. It also provides interfaces of the association mining algorithms Apriori and Eclat [69].

- lattice: It is a high-level data visualization system with an emphasis on multivariate data. It supports the creation of trellis graphs to display multivariate relationship between variables, conditioned on one or more other variables via $R$ graphics [69].

- rootSolve: It finds the root of nonlinear functions, solves the steady-state conditions for uni/multi-component and equilibrium analysis of ordinary differential equations via a dynamically running; like gradient and Jacobian matrices, non-linear equations by the Newton-Raphson algorithm.

Table 6. Tools used to evaluate and compare biclustering algorithms

\begin{tabular}{|l|l|l|}
\hline Tool & Biclustering algorithms & Reference \\
\hline Lattice & Galois lattice & {$[17]$} \\
\hline arules & rules & {$[71]$} \\
\hline rootSolve, pracma & Newton Raphson & {$[71]$} \\
\hline blockcluster & Coclustering & {$[17]$} \\
\hline biclustGUI & $\begin{array}{l}\text { CC, Plaid, BiMAX,, XMOTIFs, xQuest, } \\
\text { Spectral, FABIA, ISA }\end{array}$ & {$[20]$} \\
\hline biclust & Plaid, BiMAX, xMOTIFs, xQuest, Spectral & {$[17]$} \\
\hline BcDiag & biclust, eisa, isa2 & {$[17]$} \\
\hline FABIA, FABIAs, & FABIA & {$[40]$} \\
FABIAp, & & {$[70]$} \\
\hline NMF & NMF & {$[26]$} \\
\hline s4vd & s4vd & {$[38]$} \\
\hline qubic & Rqubic & {$[17]$} \\
\hline eisa, isa 2 & ISA & {$[72]$} \\
\hline BicARE & FLOC & {$[46]$} \\
\hline ThreeWayPlaid & Plaid for three-dimensional data & {$[44]$} \\
\hline IBBigs & iBBiG & {$[73,41]$} \\
\hline Superbiclust & Ensemble Biclustering & {$[46]$} \\
\hline HSSVD & HSSVD & {$[45]$} \\
\hline FacPad & Factor analysis for pathways & {$[74]$} \\
\hline FastICA & Fast independent component analysis & {$[75]$} \\
\hline CMonkey & cMonkey & \\
\hline & & \\
\hline
\end{tabular}

- pracma: It root finds through Newton-Raphson or Secant algorithms [70] via using functions from numerical analysis and linear algebra, numerical optimization, differential equations and some special functions. It also uses Matlab function names where appropriate to simplify porting.

- BicARE: It is based on the FLOC algorithm [23] for biclustering analysis and results exploration.

- BcDiag: It provides methods for data pre-processing, visualization, and statistical validation to diagnostic and visualize in two-dimensional data based on two way anova [40] and median polish residual plots for biclust package output obtained from biclust, eisa-isa2 and fabia packages [17][40]. In addition, the biclust package can be used via biclustGUI, i.e. $\mathrm{R}$ commander plug in. 
- blockcluster: It performs coclustering of binary, contingency and categorical datasets with utility functions to visualize the coclustered data. It contains a function cocluster which

- performs coclustering and returns object of appropriate class. It also contains coclust strategy function which returns an object of class strategy.

- rqubic: It represents an implementation of the QUBIC algorithm [38] for the qualitative biclustering with gene expression data.

- HSSVD: It discovers and compares subgroups of patients and genes which simultaneously display unusual levels of variability. It detects both mean and variance biclusters by testing the biclustering with heterogeneous variance.

- iBBig: It optimizes applying binary data analysis to meta-gene set analysis of gene expression datasets. It extracts iteratively groups of phenotypes from multiple studies that are associated with similar gene sets without requiring prior knowledge of the number or scale of clusters and allows discovery of clusters with diverse sizes.

- NMF: It provides a framework to perform Non-negative Matrix Factorization (NMF). It implements a set of already published algorithms and seeding methods, and provides a framework to test, develop and plug new/custom algorithms. It performs parallel computations on multicore machines.

- $\quad$ s4vd: It performs a biclustering via sparse singular value decomposition (svd) with a nested stability selection. The result is an biclust object and thus all methods of the biclust package can be applied.

- superbiclust: It generates as a result a number of (or super) biclusters with none or low overlap from a bicluster set, i.e. ensemble biclustering [42], with respect to the initialization parameters for a given bicluster solution. The set of robust biclusters is based on the similarity of its elements, i.e. overlap, and on the hierarchical tree obtained via cut-off points.

\section{DATASETS}

There are many microarray datasets, related to $\mathrm{R}$ package, used to evaluate biclustering algorithms [68]. Table 7. presents a few examples on these datasets.

Table 7. Microarray datasets used to evaluate biclustering algorithms

\begin{tabular}{|l|l|}
\hline Package & List of datasets \\
\hline $\begin{array}{l}\text { aroma. Copy-number } \\
\text { (n) and aroma. for } \\
\text { affyrmetrix anpuce }\end{array}$ & Spleen \\
\hline Abd & Analysis of Biological Data (abd) \\
\hline ICluster & Breast cancer, DNA cn, breast.chr17 \\
\hline ORCME & Gene expression \\
\hline Adegenet & Genetic and genomic \\
\hline SNPMClust & Dose-response microarray \\
\hline DCGL & Differential co-expression and regulation analysis \\
\hline Opmdata & OmniLog(R) Phenotype Microarray data (opmdata) \\
\hline Knorm & Across multiple biologically interrelated experiments \\
\hline Biclust & BicatYeast \\
\hline DDHFm & Data-Driven Haar-Fisz for Microarrays (DDHFm) \\
\hline integrativeMEdata & Categorical clinical factors, cancer microarray \\
\hline Madsim & Flexible microarray data simulation model (madsim) \\
\hline EMA & Easy Microarray data Analysis (EMA) \\
\hline FBN & SNP microarray \\
\hline BioConductor & Acute Lymphocytic Leukemia (ALL), arrayMissPattern. \\
\hline
\end{tabular}




\begin{tabular}{|l|l|}
\hline $\begin{array}{l}\text { Bioconductor } \\
\text { annotation } \\
\text { Data }\end{array}$ & $\begin{array}{l}\text { GO.db, GO_dbconn, GOBPANCESTOR, GOBPCHILDREN, } \\
\text { GOBPOFFSPRING, GOBPPARENTS, GOCCANCESTOR, } \\
\text { GOCCCHILDREN, GOCCOFFSPRING, GOCCPARENTS, } \\
\text { GOMAPCOUNTS, }\end{array}$ \\
\hline Lemma & Laplace approximated EM Microarray Analysis (lemma) \\
\hline Maanova & N-dye Micro 18 -array affymetrix experiment \\
\hline GeneARMA & Time-course microarray with periodic gene expression \\
\hline iGenomicViewer & IGGVex \\
\hline CLAG & Breast tumor cells \\
\hline
\end{tabular}

\section{CONCLUSION}

The biclustering of microarray data has been the subject of a large research. No one of the existing biclustering algorithms is perfect. The construction of biologically significant groups of biclusters for large microarray data is still a problem that requires a continuous work. Biological validation of biclusters of microarray data is one of the most important open issues. So far, there are no general guidelines in the literature on how to validate biologically extracted biclusters.

\section{REFERENCES}

[1] Ouafae Kaissi. Analyse de Données Transcriptomiques pour La Recherche de Biomarqueurs Liés à Certaines Pathologies Cancéreuses. PhD thesis, University Abdelmalek Essaadi, Tangier, Morocco, sep 2014.

[2] Sara C. Madeira and Arlindo L. Oliveira. A polynomial time biclustering algorithm for finding approximate expression patterns in gene expression time series. Algorithms for Molecular Biology, 4(8), June 2009.

[3] W. Ayadi and M. Elloumi. Algorithms in Computational Molecular Biology : Techniques, Approaches and Applications. chapter Biclustering of Microarray Data, 2011.

[4] Sara C. Madeira and Arlindo L. Oliveira. Biclustering algorithms for biological data analysis: A survey. IEEE/ACM Trans. Comput. Biol. Bioinformatics, 1:24-45, 2004. ISSN 1545-5963.

[5] Law Ngai-Fong Siu Wan-Chi Cheng, Kin-On and Alan Wee-Chung. Identification of coherent patterns in gene expression data using an efficient biclustering algorithm and parallel coordinate visualization. BMC Bioinformatics, 2008.

[6] Xiaowen Liu and Lusheng Wang. Computing the maximum similarity bi-clusters of gene expression data. Bioinformatics, 23(1):50-56, 2007.

[7] Aguilar-Ruiz and Jesús S. Shifting and scaling patterns from gene expression data. Bioinformatics, 21(20): 3840-3845, 2005.

[8] Hyuk Cho and Inderjit S. Dhillon. Coclustering of human cancer microarrays using minimum sumsquared residue coclustering. IEEE/ACM Trans. Comput. Biol. Bioinformatics, 5(3):385-400, 2008.

[9] Ranajit Das and al. Evolutionary biclustering with correlation for gene interaction networks. In Pattern Recognition and Machine Intelligence, Second International Conference, PReMI 2007, Kolkata, India, December 18-22, 2007, Proceedings, pages 416-424, 2007.

[10] Yizong Cheng and George M. Church. Biclustering of expression data. pages 93-103, 2000.

[11] Li Teng and Laiwan Chan. Discovering biclusters by iteratively sorting with weighted correlation coefficient in gene expression data. Signal Processing Systems, 50:267-280.

[12] Carazo J.-Kochi K. Lehmann-D. Pascual-Montano, A. and R. D. Pascual-Marqui. Nonsmooth nonnegative matrix factorization (nsnmf). IEEE, 2006.

[13] Rodrigo Santamara, Roberto Theran, and Luis Quintales. Bicoverlapper: A tool for bicluster visualization. Bioinformatics, 24:1212-1213, 2008.

[14] Roberto Therón Rodrigo Santamaría and Luis Quintales. A visual analytics approach for understanding biclustering results from microarray data. BMC Bioinformatics, 9(247), 2008.

[15] Pinheiro M. Arrais-J. Gomes A. C. Carreto L. Freitas A. Oliveira J. L. Moura, G. and M. A. Santos. Large scale comparative codon-pair context analysis unveils general rules that fine-tune evolution of mrna primary structure. PLoS ONE., 2007.

[16] Yuval Kluger, Ronen Basri, Joseph T. Chang, and Mark Gerstein. Spectral biclustering of microarray cancer data: Co-clustering genes and conditions. Genome Research, 13:703-716, 2003. 
[17] Santamaria R. Khamiakova-T. Sill M. Theron R. Quintales L. Kaiser, S. and F. Leisch. biclust: Bicluster algorithms. R package., 2011.

[18] Eugenio Cesario Fabrizio Angiulli and Clara Pizzuti. Random walk biclustering for microarray data. Information Sciences, 178(6):1479-1497, 2008.

[19] Elloumi M. Ayadi, W. and J.-K. Hao. Bicfinder: a biclustering algorithm for microarray data analysis. Knowledge and Information Systems., 2012.

[20] Jan Ihmels, Sven Bergmann, and Naama Barkai. Defining transcription modules using large-scale gene expression data. Bioinformatics, 20(13):1993-2003, 2004.

[21] Chor B.-Karp R. Ben-Dor, A. and Z. Yakhini. Clustering gene expression patterns. 6, 2002.

[22] Amos Tanay, Roded Sharan, and Ron Shamir. Discovering statistically significant biclusters in gene expression data. In In Proceedings of ISMB 2002, pages 136-144, 2002.

[23] Jiong Yang and al. Enhanced biclustering on expression data.

[24] Chor Benny Karp Richard Ben-Dor, Amir. and Zohar. Yakhini. Discovering local structure in gene expression data: The order-preserving submatrix problem. In Proceedings of the Sixth Annual International Conference on Computational Biology, RECOMB '02, pages 49-57, New York, NY, USA, 2002. ACM.

[25] Hossam S. Sharara and Mohamed A. Ismail. Bisoft: A semi-fuzzy approach for biclustering gene expression data. In BIOCOMP, 2008.

[26] Martin Sill, Sebastian Kaiser, Axel Benner, and Annette Kopp-Schneider. Robust biclustering by sparse singular value decomposition incorporating stability selection. Bioinformatics, 27:2089-2097, 2011.

[27] Miranda van Uitert, Wouter Meuleman, and Lodewyk F. A. Wessels. Biclustering sparse binary genomic data. Journal of Computational Biology, 15(10):1329-1345, 2008.

[28] Perez-Pulido A. J. Rodriguez-Baena, D. S. and J.S. Aguilara-Ruiz. A biclustering algorithm for extracting bit-patterns from binary datasets. Bioinformatics., 2011.

[29] Elloumi M. Ayadi, W. and J.-K. Hao. A biclustering algorithm based on a bicluster enumeration tree: application to dna microarray data. BioData Mining., 2009.

[30] Tze-Haw Huang ; XingXing Song ; Mao Lin Huang. Optimized data acquisition by time series clustering in opc. IEEE., 2011.

[31] Inderjit S. Dhillon, Subramanyam Mallela, and Dharmendra S. Modha. Information-theoretic coclustering. In Proceedings of the ninth ACM SIGKDD international conference on Knowledge discovery and data mining, pages 89-98. ACM Press, 2003.

[32] Jiun-Rung Chen and Ye-In Chang. A condition-enumeration tree method for mining biclusters from dna microarray data sets. Elsevier, 97:44-59, 2007.

[33] Stefan Bleuler Oliver Voggenreiter and Wilhelm Gruissem. Exact biclustering algorithm for the analysis of large gene expression data sets. Eighth International Society for Computational Biology (ISCB) Student Council Symposium Long Beach, CA, USA.July, pages 13-14, 2012.

[34] Joana P. Gonalves and Sara C. Madeira. e-bimotif: Combining sequence alignment and biclustering to unravel structured motifs. In IWPACBB, volume 74, pages 181-191, 2010.

[35] Shamir and al. Expander - an integrative program suite for microarray data analysis. BMC Bioinformatics, 6: 232, 2005.

[36] Dong Wang and al. Mapping query to semantic concepts: Leveraging semantic indices for automatic and interactive video retrieval. In ICSC '07: Proceedings of the International Conference on Semantic Computing, pages 313-320, 2007.

[37] W. Ahmad. chawk: An efficient biclustering algorithm based on bipartite graph crossing minimization. 2007.

[38] Haibao Tang Andrew H. Paterson Guojun Li, Qin Ma and Ying Xu. Qubic: a qualitative biclustering algorithm for analyses of gene expression data. 2009.

[39] Nir Friedman, Lise Getoor, Daphne Koller, and Avi Pfeffer. Learning probabilistic relational models. In IJCAI, pages 1300-1309, 1999.

[40] Sepp Hochreiter, Ulrich Bodenhofer, Martin Heusel, Andreas Mayr, Andreas Mitterecker, Adetayo Kasim, Tatsiana Khamiakova, Suzy Van Sanden, Dan Lin 0004, Willem Talloen, Luc Bijnens, Hinrich W. H. Göhlmann, Ziv Shkedy, and Djork-Arné Clevert. Fabia: factor analysis for bicluster acquisition. Bioinformatics, 26(12):1520-1527, 2010.

[41] Mohamed Nadif and Gérard Govaert. Block clustering via the block gem and two-way em algorithms. In AICCSA'05, pages -1-1, 2005.

[42] Mohamed Nadif and Gerard Govaert. A comparison between block cem and two-way cem algorithms to cluster a contingency table. In PKDD’05, pages 609-616, 2005. 
[43] Baocheng W. Guifen, C. and Y. Helong. The implementation of parallel genetic algorithm based on matlab. Advanced Parallel Processing Technologies., 2007.

[44] Daniel Gusenleitner, Eleanor Howe, Stefan Bentink, John Quackenbush, and Aedin C. Culhane. ibbig: iterative binary bi-clustering of gene sets. Bioinformatics, 28(19):2484-2492, 2012.

[45] Lazzeroni and Owen. Plaid models for gene expression data. Statistica Sinica., 2002.

[46] Shawn Mankad and George Michailidis. Biclustering three-dimensional data arrays with plaid models. Journal of Computational and Graphical Statistics, 2013.

[47] Ole Andreatta, Massimo Lund and Morten Nielsen. Simultaneous alignment and clustering of peptide data using a gibbs sampling approach. Bioinformatics, 29(1):8-14, 2013.

[48] Hartigan. Clustering Algorithms, chapter Direct splitting. 1975.

[49] Gerard GOVAERT. La classification croisee. Modulad, 1983.

[50] Wunsch II Xu, Rui and Donald C. Bartmap: A viable structure for biclustering. Neural Netw., 24:709-716, September, 2011.

[51] Douglas Creighton Saeid Nahavandi. Thanh Nguyen, Abbas Khosravi. Spike sorting using locality preserving projection with gap statistics and landmark-based spectral clustering. Neuroscience Methods., 2014.

[52] I. Llatas, A.J. Quiroz, and J.M. Renom. A fast permutation-based algorithm for block clustering. Test, 6(2): 397-418, 1997.

[53] G. Govaert and M. Nadif. Co-Clustering. FOCUS Series. Wiley, 2013.

[54] G. Getz, E. Levine, and E. Domany. Coupled two-way clustering analysis of gene microarray data. Proc. Natl. Acad. Sci. USA, 97:12079-12084, 2000.

[55] Amela Preli'c, Stefan Bleuler, Philip Zimmermann, Anja Wille, Peter Bühlmann, Wilhelm Gruissem, Lars Hennig, Lothar Thiele, and Eckart Zitzler. A systematic comparison and evaluation of biclustering methods for gene expression data. Bioinformatics, 22:1122-1129, 2006.

[56] J. Caldas and S. Kaski. Hierarchical generative biclustering for microrna expression analysis. Computational Biology., 2011.

[57] M. Charrad. Une approche gnrique pour l-analyse croisant contenu et usage des sites web par des methodes de bipartitionnement. PhD thesis, Paris and ENSI, University of Manouba, 2010.

[58] Yves Lechevallier Malika Charrad, Gilbert Saporta, and Mohamed Ben Ahmed. Determination du nombre des classes dans l'algorithme croki de classification croisee. In EGC'09, pages 447-448, 2009.

[59] Stanislav Busygin and al. Double conjugated clustering applied to leukemia microarray data. 2002.

[60] Khalid Benabdeslem and Kais Allab. Bi-clustering continuous data with self-organizing map. Neural Computing and Applications, 22(7):1551-1562, 2013.

[61] Chun Tang, Li Zhang 0008, Aidong Zhang, and Murali Ramanathan. Interrelated two-way clustering: An unsupervised approach for gene expression data analysis. pages 41-48, 2001.

[62] Eleni Mina. Applying biclustering to understand the molecular basis of phenotypic diversity. Phd. Utrecht University Faculty of Science Department of Information and Computing Sciences, 2011.

[63] Akdes Serin. Biclustering analysis for large scale data. Phd., 2011.

[64] Michael Ashburner. Gene ontology: tool for the unification of biology. Nature Genetics 25, pages 25 $-29,2000$.

[65] Gene ontology consortium. Internet:, . URL http://www.geneontology.org/,note= September2014.

[66] Pietro Hiram Guzzi, Marianna Milano, and Mario Cannataro. Mining association rules from gene ontology and protein networks: Promises and challenges. Procedia Computer Science, 29(0):1970 1980, 2014. International Conference on Computational Science.

[67] Xuebo Song, Lin Li, Pradip K. Srimani, Philip S. Yu, and James Z.Wang. Measure the semantic similarity of go terms using aggregate information content. IEEE/ACM Trans. Comput. Biol. Bioinformatics, 11:468-476, 2014.

[68] Cran package. Internet:, . URL http://cran.r-project.org/web/packages. July 2014.

[69] Kuznetsov S. O. Macko J. Jr. W. M. Kaytoue, M. and A. Napoli. Mining biclusters of similar values with triadic concept analysis. The Eighth International Conference on Concept Lattices and Their Applications., 2011.

[70] Chris H. Q. Ding, Tao Li, and Wei Peng. Nonnegative matrix factorization and probabilistic latent semantic indexing: Equivalence chi-square statistic, and a hybrid method. In AAAI'06, 2006.

[71] Haifa BenSaber. Classification non supervisiee des donnees des puces a ADN", ESSTT. 2010.

[72] Jiong Yang, HaixunWang,WeiWang 0010, and Philip S. Yu. An improved biclustering method for analyzing gene expression profiles. International Journal on Artificial Intelligence Tools, 14(5):771790, 2005. 
[73] Mehmet Koyuturk. Using protein interaction networks to understand complex diseases. Computer, 45(3): 31-38, 2012.

[74] C Heaton J L Marchini and B D Ripley. fastica: Fastica algorithms to perform ica and projection pursuit. R package, 2013.

[75] Baliga N. S. Reiss, D. J. and Bonneau. cmonkey integrated biclustering algorithm. R package, 2012.

\section{AUTHORS}

Professor ELLOUMI Mourad : Full Professor in Computer Science Head of the BioInformatics Group (BIG) of The Laboratory of Technologies of Information and Communication, and Electrical Engineering (LaTICE), National High School of Engineers of T unis (ENSIT), University of Tunis, Tunisia, and Professor at the Faculty of Economic Sciences and Management of Tunis (FSEGT), University of Tunis El Manar, Tunisia.

Mrs BEN SABER Haifa : Phd student on the BioInformatics Group (BIG) of The Laboratory of Technologies of Information and Communication, and Electrical Engineering (LaTICE), National High School of Engineers of Tunis (ENSIT), University of Tunis, Tunisia, and Assistant at the Time Université, Tunisia.
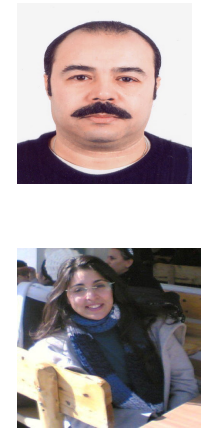